\title{
Sputnik Tracker: Having a Companion Improves Robustness of the Tracker
}

\author{
Lukáš Cerman, Jiří Matas, and Václav Hlaváč \\ Czech Technical University \\ Faculty of Electrical Engineering, Center for Machine Perception \\ 12135 Prague 2, Karlovo naměstí 13, Czech Republic \\ \{cermal1,hlavac\}@fel.cvut.cz, matas@cmp.felk.cvut.cz
}

\begin{abstract}
Tracked objects rarely move alone. They are often temporarily accompanied by other objects undergoing similar motion. We propose a novel tracking algorithm called Sputnil Tracker. It is capable of identifying which image regions move coherently with the tracked object. This information is used to stabilize tracking in the presence of occlusions or fluctuations in the appearance of the tracked object, without the need to model its dynamics. In addition, Sputnik Tracker is based on a novel template tracker integrating foreground and background appearance cues. The time varying shape of the target is also estimated in each video frame, together with the target position. The time varying shape is used as another cue when estimating the target position in the next frame.
\end{abstract}

\section{Introduction}

One way to approach the tracking and scene analysis is to represent an image as a collection of independently moving planes [12/34]. One plane (layer) is assigned to the background, the remaining layers are assigned to the individual objects. Each layer is represented by its appearance and support (segmentation mask). After initialization, the motion of every layer is estimated in each step of the video sequence together with the changes of its appearance and support.

The layer-based approach has found its applications in video insertion, sprite-based video compression, and video summarization [2]. For the purpose of a single object tracking, we propose a similar method using only one foreground layer attached to the object and one background layer. Other objects, if present, are not modelled explicitly. They become parts of the background outlier process. Such approach can be also viewed as a generalized background subtraction combined with an appearance template tracker.

Unlike background subtraction based techniques [5|6778], which model only background appearance, or appearance template trackers, which usually model only the foreground appearance [9]10 11/12], the proposed tracker uses the complete observation model which makes it more robust to appearance changes in both foreground and background.

The image-based representation of both foreground and background, inherited from the layer-based approaches, contrasts with statistical representations used by classifiers [13] or discriminative template trackers [1415], which do not model the spatial structure of the layers. The inner structure of each layer can be useful source of information for localizing the layer.

\footnotetext{
${ }^{1}$ Sputnik, pronounced \'sput-nik in Russian, was the first Earth-orbiting satellite launched in 1957. According to Merriam-Webster dictionary, the English translation of the Russian word sputnik is a travelling companion.
}

A.-B. Salberg, J.Y. Hardeberg, and R. Jenssen (Eds.): SCIA 2009, LNCS 5575, pp. 291-300, 2009.

(C) Springer-Verlag Berlin Heidelberg 2009 

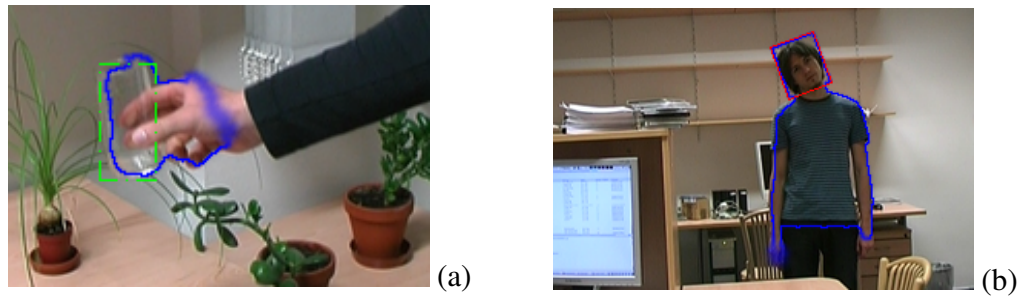

Fig. 1. Objects with a companion. Foreground includes not just the main object, e.g., (a) a glass or (b) a head, but also other image regions, such as (a) hand or (b) body.

The foreground layer often includes not just the object of interest but also other image regions which move coherently with the object. The connection of the object to the companion may be temporary, e.g., a glass can be picked up by hand and dragged from the table, or it may be permanent, e.g., a head of a man always moves together with his torso, see Figure 1 for examples. As the core contribution of this paper, we show how the companion, i.e., the non-object part of the foreground motion layer, contributes to robust tracking and expands situations in which successful tracking is possible, e.g, when the object of interest is not visible or abruptly changes its appearance. Such situations would distract the trackers that look only for the object itself.

The task of tracking a single object can be then decomposed to several sub-problems: (1) On-line learning of the foreground layer appearance, support and motion, i.e., "What is the foreground layer?". (2) Learning of the background layer appearance, support and motion. In our current implementation, the camera is fixed and the background appearance is learned off-line from the training sequence. However, the principle of the proposed tracker allows us to estimate the background motion and its appearance changes on-line in the future versions. (3) Separating the object from its companion, i.e., "Where is the object?". (4) Modelling appearance of the object.

The proposed Sputnik Tracker is based on this reasoning. It learns and is able to estimate which parts of the image area accompany the object, be it temporarily or permanently, and which parts together with the object form the foreground layer. In this paper we do not deal with tracker initialization and re-initialization after failure.

The Sputnik Tracker requires the foreground to be modelled as a structure of connected, independently moving parts, unlike approaches based on the pictorial structures [7|16 17]. Theforegroundlayeris represented by a plane containing only image regions which perform similar movement. To track a part of an object, the Sputnik Tracker does not need to have a prior knowledge of the object structure, i.e., the number of parts and their connections.

The rest of the paper is structured as follows: In Section 2, the probabilistic model implemented in Sputnik Tracker will be explained together with the on-line learning of the model parameters. The tracking algorithm will be described. In Section 3 , it will be demonstrated on several challenging sequences how the estimated companion contributes to robust tracking. The contributions will be concluded in Section 4

\section{The Sputnik Tracker}

\subsection{Integrating Foreground and Background Cues}

We pose the object tracking probabilistically as finding the foreground position $l^{\star}$, in which the likelihood of the observed image $I$ is maximized over all possible locations $l$ given the foreground model $\phi_{F}$ and the background model $\phi_{B}$ 


$$
l^{\star}=\underset{l}{\operatorname{argmax}} P\left(I \mid \phi_{F}, \phi_{B}, l\right) .
$$

When the foreground layer has the position $l$ then the observed image can be divided in two disjoint areas $-I_{F(l)}$ containing pixels associated with foreground layer and $I_{B(l)}$ containing pixels belonging to the background layer. Assuming that pixel intensities observed on the foreground are independent of those observed on the background, the likelihood of observing the image $I$ can be rewritten as

$$
P\left(I \mid \phi_{F}, \phi_{B}, l\right)=P\left(I_{F(l)}, I_{B(l)} \mid \phi_{F}, \phi_{B}\right)=P\left(I_{F(l)} \mid \phi_{F}\right) P\left(I_{B(l)} \mid \phi_{B}\right) .
$$

Ignoring dependencies on the foreground-background boundary:

$$
P\left(I \mid \phi_{B}\right)=P\left(I_{F(l)} \mid \phi_{B}\right) P\left(I_{B(l)} \mid \phi_{B}\right),
$$

Equation (2) can be rewritten as

$$
P\left(I \mid \phi_{F}, \phi_{B}, l\right)=\frac{P\left(I_{F(l)} \mid \phi_{F}\right)}{P\left(I_{F(l)} \mid \phi_{B}\right)} P\left(I \mid \phi_{B}\right) .
$$

The last term in Equation (4) does not depend on $l$. It follows that likelihood of the whole image (with respect to $l$ ) is maximized by maximizing the likelihood ratio of the image region $I_{F(l)}$ with the respect to the foreground $\phi_{F}$ and background model $\phi_{B}$. The optimal position $l^{\star}$ is then

$$
l^{\star}=\underset{l}{\operatorname{argmax}} \frac{P\left(I_{F(l)} \mid \phi_{F}\right)}{P\left(I_{F(l)} \mid \phi_{B}\right)} .
$$

Note that by modelling $P\left(I_{F(l)} \mid \phi_{B}\right)$ as the uniform distribution with respect to $I_{F(l)}$, one gets, as a special case, a standard template tracker which maximizes likelihood of $I_{F(l)}$ with respect to the foreground model only.

\subsection{Object and Companion Models}

Very often some parts of the visible scene undergo the same motion as the object of interest. The foreground layer, the union of such parts, is modelled by the companion model $\phi_{C}$. The companion model is adapted on-line in each step of tracking. It is gradually extended by the neighboring image areas which exhibit the same movement as the tracked object. The involved areas are not necessarily connected.

Should such a group of objects split later, it must be decided which image area contains the object of interest. Sputnik Tracker maintains another model for this reason, the object model $\phi_{O}$, which describes the appearance of the main object only. Unlike the companion model $\phi_{C}$, which adapts on-line very quickly, the object model $\phi_{O}$ adapts slowly, with lower risk of drift.

In the current implementation, both models are based on the same pixel-wise representation:

$$
\begin{aligned}
& \phi_{C}=\left\{\left(\mu_{j}^{C}, s_{j}^{C}, m_{j}^{C}\right) ; j \in\{1 \ldots N\}\right\}, \\
& \phi_{O}=\left\{\left(\mu_{j}^{O}, s_{j}^{O}, m_{j}^{O}\right) ; j \in\left\{1 \ldots N_{O}\right\}\right\},
\end{aligned}
$$



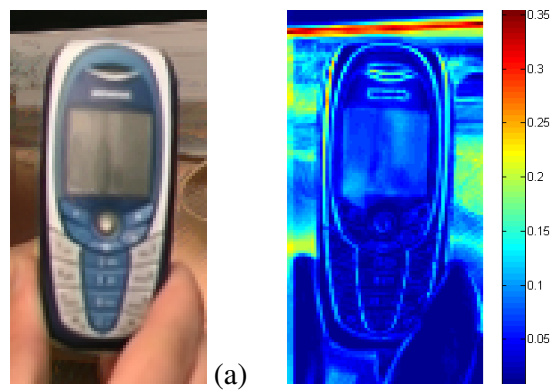

(b)
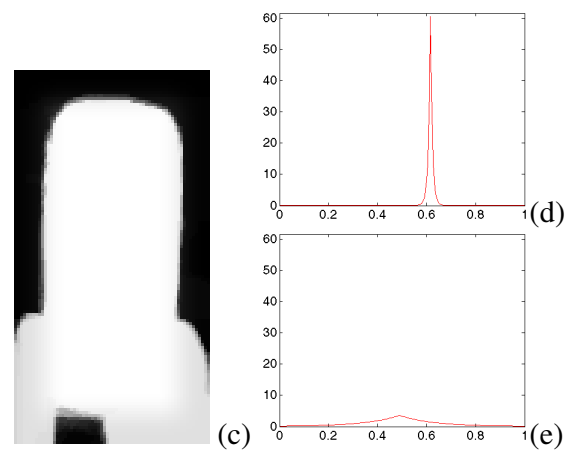

Fig. 2. Illustration of the model parameters: (a) median, (b) scale and (c) mask. Right side displays the pixel intensity PDF which is parametrized by its median and scale, see Equation (8) and (9). There are two examples, one of pixel with (d) low variance and other with (e) high variance.

where $N$ and $N_{O}$ denote the number of pixels in the template, which is illustrated in Figure 2 In the probabilistic model, each individual pixel is represented by the probability density function (PDF) based on the mixture of Laplace distribution

$$
f(x \mid \mu, s)=\frac{1}{2 s} \exp \left(-\frac{|x-\mu|}{s}\right)
$$

restricted to the interval $\langle 0,1\rangle$, and uniform distribution over the interval $\langle 0,1\rangle$ :

$$
p(x \mid \mu, s)=\omega U_{\langle 0,1\rangle}(x)+(1-\omega) f_{\langle 0,1\rangle}(x \mid \mu, s),
$$

where $U_{\langle 0,1\rangle}(x)=1$ represents the uniform distribution and

$$
f_{\langle 0,1\rangle}(x \mid \mu, s)=\left[f(x \mid \mu, s)+\frac{\int_{\langle 0,1\rangle} f\left(x^{\prime} \mid \mu, s\right) \mathrm{d} x^{\prime}}{\iint_{\langle 0,1\rangle} 1 \mathrm{~d} x^{\prime}}\right]
$$

represents the restricted Laplace distribution. The parameter $\omega \in(0,1)$ weighs the mixture. It has the same value for all pixels and represents the probability of an unexpected measurement. The individual pixel PDFs are parametrized by their median $\mu$ and scale $s$.

The mixture of the Laplace distribution with the uniform distribution provides distribution with heavier tails which is more robust to unpredicted disturbances. Examples of PDF in the form of Equation (9) are shown in Figure 2d,e. The distribution in the form of Equation (10) has the desirable property that it approaches uniform distribution by increasing the uncertainty in the model. This is likely to happen in fast and unpredictably changing object areas that would otherwise disturb the tracking.

The models $\phi_{C}$ and $\phi_{O}$ also include segmentation mask (support) which assigns each pixel $j$ in the model the value $m_{j}$ representing a probability that the pixel belongs to the object.

\subsection{Evolution of the Models}

At the end of each tracking step at time $t$, after the new position of the object has been estimated, the model parameters $\mu, s$ and the segmentation mask are updated. For each pixel in the model, its median is updated using the exponential forgetting principle, 


$$
\mu^{(t)}=\alpha \mu^{(t-1)}+(1-\alpha) x
$$

where $x$ is the observed intensity of the corresponding image pixel in the current frame and $\alpha$ is the parameter controlling the speed of exponential forgetting. Similarly, the scale is updated as

$$
s^{(t)}=\max \left\{\alpha s^{(t-1)}+(1-\alpha)\left|x^{(t)}-\mu^{(t)}\right|, s_{\min }\right\} .
$$

The scale values are limited by the manually chosen lower bound $s_{\min }$ to prevent overfitting and to enforce robustness to a sudden change of the previously stable object area.

The segmentation mask of the companion model $\phi_{C}$ is updated at each step of the tracking following updates of $\mu$ and $s$. First, a binary segmentation $A=\left\{a_{j} ; a_{j} \in\right.$ $\{0,1\}, j \in 1 \ldots N\}$ is calculated using Graph Cuts algorithm [18]. An update to the object segmentation mask is then obtained as

$$
m_{j}^{C,(t)}=\alpha m_{j}^{C,(t-1)}+(1-\alpha) a_{j}
$$

\subsection{Background Model}

The background is modelled using the same distribution as the foreground. Background pixels are considered independent and are represented by PDF expressed by formula (9). Each pixel of the background is then characterized by its median $\mu$ and scale $s$ :

$$
\phi_{B}=\left\{\left(\mu_{i}^{B}, s_{i}^{B}\right) ; i \in\{1 \ldots\|I\|\}\right\},
$$

This model is suitable for a fixed camera. However, by geometrically registering consecutive frames in the video sequence, it might be used with pan-tilt-zoom (PTZ) cameras, which have a lot of applications in surveillance, or even with freely moving camera, provided that the movement is not large so that the robust tracker will overcome the model error caused by the change of the parallax. Cases with almost planar background, like aerial images of the Earth surface, can be also handled by the rigid geometrical image registration.

In the current implementation, the background parameters $\mu$ and scale $s$ are learned off-line from a training sequence using EM algorithm. The sequence does not necessarily need to exhibit empty scene. It might also contain objects moving in the foreground. The foreground objects are detected as outliers and are robustly filtered out by the learning algorithm. Description of the learning algorithm is out of the scope of this paper.

\subsection{The Tracking Algorithm}

The state of the tracker is characterized by object appearance model $\phi_{O}$, companion model $\phi_{C}$ and object location $l$. In the current implementation, we model the affine rigid motion of the object. This does not restrict us to track rigid objects only, it only limits the space of possible locations $l$ such that the coordinate transform $j=\psi(i \mid l)$ is affine. The transform maps indices $i$ in the image pixel to indices $j$ in the model, see Figure 3 Appearance changes due to a non-rigid object or its non-affine motion are handled by adapting on-line the companion appearance model $\phi_{C}$.

The tracker is initialized by marking the area covered by the object to be tracked in the first image of the sequence. The size of the companion model $\phi_{C}$ is set to cover a 


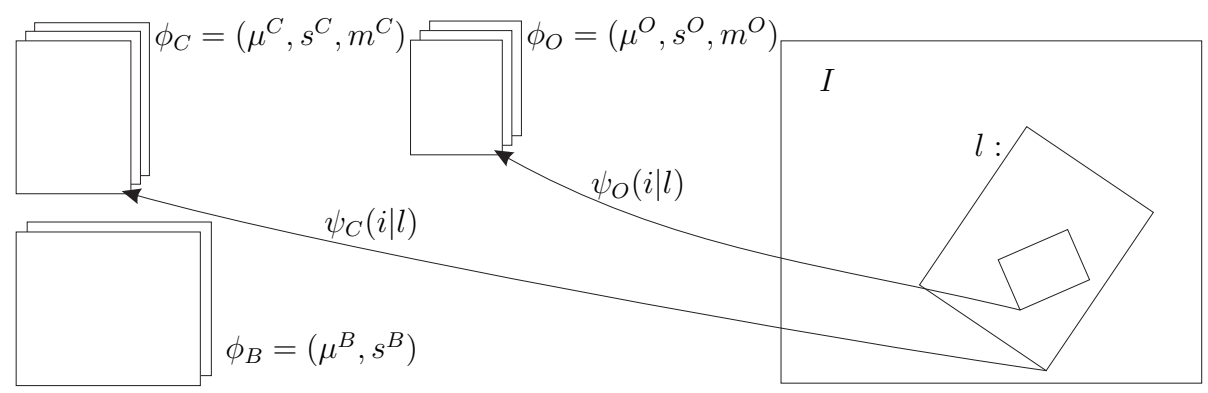

Fig. 3. Transforms between image and model coordinates

rectangular area larger than the object. That area has potential to become a companion of the object. Initial values of $\mu_{j}^{C}$ are set to image intensities observed in the corresponding image pixels, $s_{j}^{C}$ are set to $s_{\min }$. Mask values $m_{j}^{C}$ are set to 1 in areas corresponding to the object and to 0 elsewhere.

Object model $\phi_{O}$ is initialized in the similar way, but it covers only the object area. Only the scale of the object model, $s_{j}^{O}$, is updated during tracking.

Tracking is approached as minimization of the cost based on the negative logarithm of the likelihood ratio, Equation (5),

$$
\left.\mathcal{C}(l, M)=-\sum_{i \in F(l)} p\left(I(i) \mid \mu_{\psi_{M}(i \mid l)}^{M}, s_{\psi_{M}(i \mid l)}^{M}\right)+\sum_{i \in F(l)} p\left(I(i) \mid \mu_{i}^{B}, s_{i}^{B}\right)\right],
$$

where $F(l)$ are indices of image pixels covered by the object/companion if it were at the location $l$, the assignment is determined by the model segmentation mask and $\psi_{M}(i \mid l)$. The model selector (companion or object) is denoted $M \in\{O, C\}$. The following steps are executed for each image in the sequence.

1. Find the optimal object position induced by the companion model by minimizing the cost $l_{C}^{\star}=\operatorname{argmin} \mathcal{C}(l, C)$. The minimization is performed using the gradient descent method starting at the previous location.

2. Find the optimal object position induced by the object model $l_{O}^{\star}=$ $\operatorname{argmin} \mathcal{C}(l, O)$ using the same approach.

3. If $\mathcal{C}\left(l_{O}^{\star}, O\right)$ is high then continue from step 5

4. If the location $l_{O}^{\star}$ gives better fit to the object model, $\mathcal{C}\left(l_{O}^{\star}, O\right)<\mathcal{C}\left(l_{C}^{\star}, O\right)$, then set the new object location to $l=l_{O}^{\star}$ and continue from step 6 .

5. The object may be occluded or its appearance may be changed. Set the new object location to $l=l_{C}^{\star}$.

6. Update model parameters $\mu_{j}^{C}, s_{j}^{C}, m_{j}^{C}$ and $s_{j}^{O}$ using method described in Section 2.3

The above described algorithm is controlled by several manually chosen parameters which were described in the previous sections. To recapitulate, those are: $\omega-$ the probability of unexpected pixel intensity, it controls the amount of uniform distribution in the mixture PDF; $\alpha$ - the speed of the exponential forgetting; $s_{\min }$ the lover bound on the scale $s$. The unoptimized MATLAB implementation of the process takes 1 to 10 seconds per image on a standard PC. 


\section{Results}

To show the strengths of the Sputnik Tracker, a successful tracking on some challenging sequences will be demonstrated. In all following illustrations, the red rectangle is used
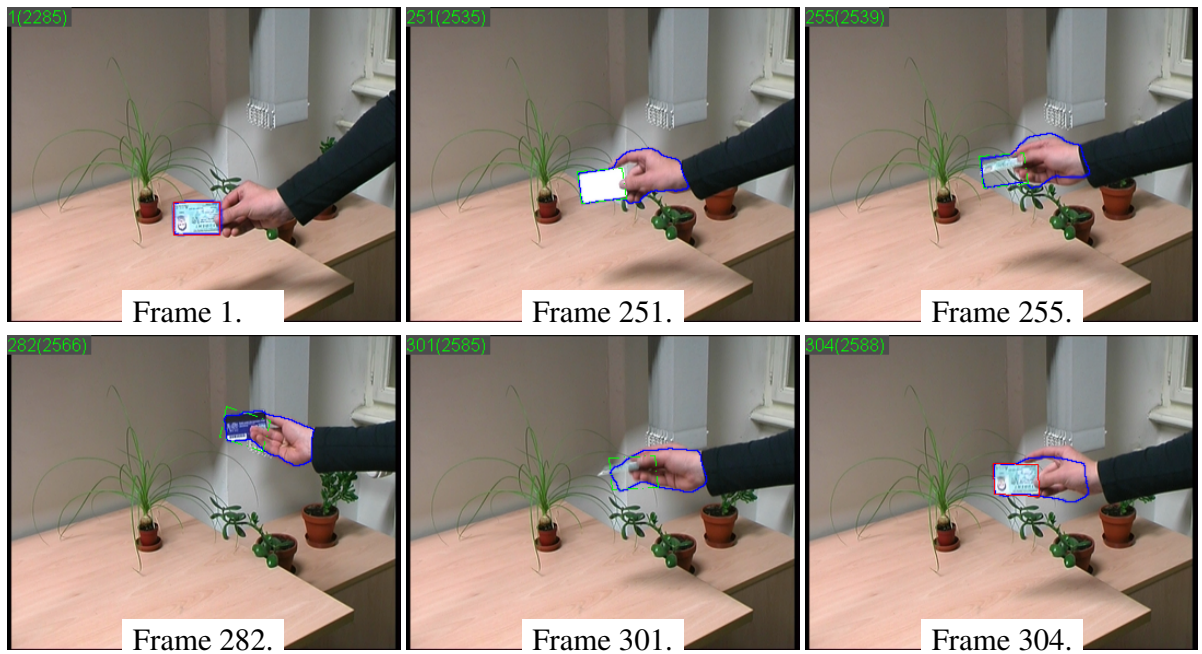

Fig. 4. Tracking a card carried by the hand. The strong reflection in frame 251 or flipping the card later does not cause the Sputnik Tracker to fail.

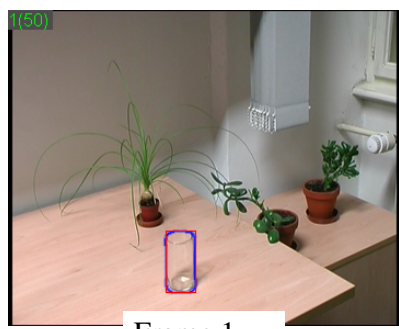

Frame 1.

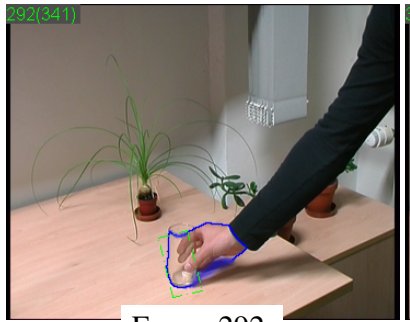

Frame 292

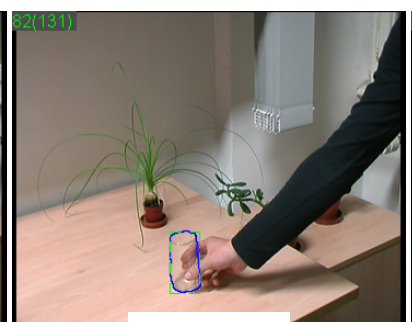

Frame 82.

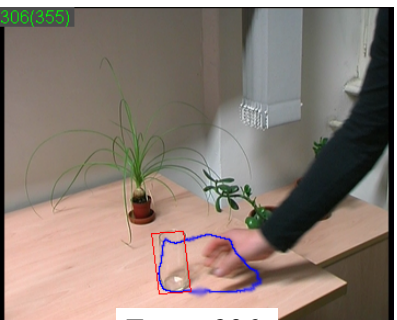

Frame 306.

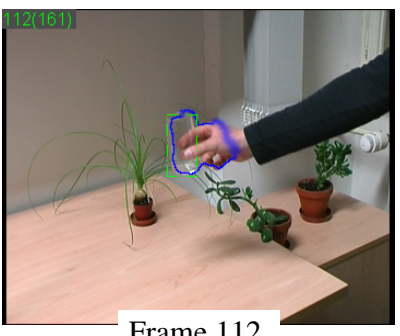

Frame 112.

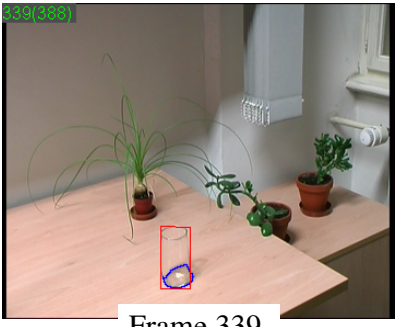

Frame 339.

Fig. 5. Tracking a glass after being picked by a hand and put back later. The glass moves with the hand which is recognized as companion and stabilizes the tracking. 

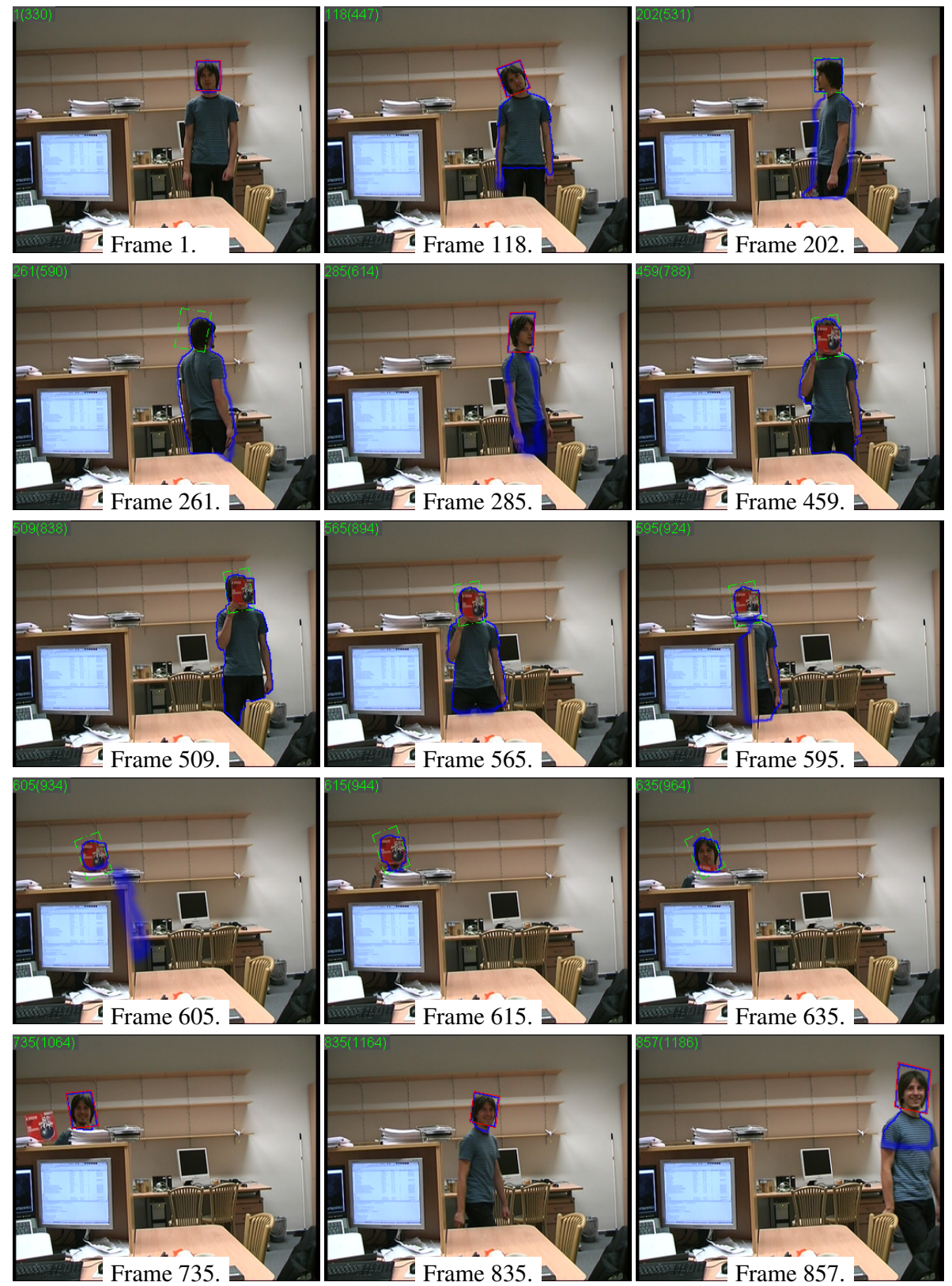

Fig. 6. Tracking the head of a man. The body is correctly recognized as a companion (the blue line). This helped to keep tracking the head while the man turns around between frames 202 and 285 and after the head gets covered with a picture in the frame 495 and the man hides behind the sideboard. In those moments, an occlusion was detected, see the green rectangle in place of the red one, but the head position was still tracked, given the companion. 
to illustrate a successful object detection, a green rectangle corresponds to the recognized occlusion or the change of object appearance. The blue line shows the contour of the foreground layer including the estimated companion. The thickness of the line is proportional to the uncertainty in the layer segmentation. The complete sequences can be downloaded from http://cmp.felk.cvut.cz/ cermal1/supplements-scia/as video files.

The first sequence shows the tracking of an ID card, see Figure 4 for several frames selected from the sequence. After initialization with the region belonging to the card, the Sputnik Tracker learns that the card is accompanied by the hand. This prevents it from failing in the frame 251 where the card reflects strong light source and its image is oversaturated. Any tracker that looks only for the object itself would have a very hard time at this moment. Similarly, the knowledge of the companion helps to keep a successful tracking even when the card is flipped in the frame 255. The appearance on the backside differs from the frontside. The tracker recognizes this change and reports an occlusion. However, the rough position of the card is still maintained with respect to the companion. When the card is flipped back it is redetected in the frame 304.

Figure 5 shows tracking of a glass being picked by a hand in the frame 82 . At this point, the tracker reports an occlusion that is caused by the fingers and the hand is becoming a companion. This allows the tracking of the glass while it is being carried around the view. The glass is dropped back to the table in the frame 292 and when the hand moves away it is recognized back in the frame 306.

Figure 6 shows head tracking through occlusion. After initialization to the head area in the first image, the Sputnik Tracker estimates the body as a companion, see frame 118. While the man turns around between frames 202 and 285 the tracker reports occlusion of the tracked object (head) and maintains its position relative to the companion. The tracking is not lost even when the head gets covered with a picture and the man moves behind a sideboard and only the picture covering the head remains visible. This would be very difficult to achieve without learning the companion. After the picture is removed in the frame 635 , the head is recognized again in the frame 735 . The man then leaves the view while his head is still being successfully tracked.

\section{Conclusion}

We have proposed a novel approach to tracking based on the observation that objects rarely move alone and their movement can be coherent with other image regions. Learning which image regions move together with the object can help to overcome occlusions or unpredictable changes in the object appearance.

To demonstrate this we have implemented a Sputnik Tracker and presented a successful tracking in several challenging sequences. The tracker learns on-line which image regions accompany the object and maintain an adaptive model of the companion appearance and shape. This makes it robust to situations that would be distractive to trackers focusing only on the object alone.

\section{Acknowledgments}

The authors wish to thank Libor Špaček for careful proofreading. The authors were supported by Czech Ministry of Education project $1 \mathrm{M} 0567$ and by EC project ICT-215078 DIPLECS. 


\section{References}

1. Tao, H., Sawhney, H.S., Kumar, R.: Dynamic layer representation with applications to tracking. In: Proceedings of the International Conference on Computer Vision and Pattern Recognition, vol. 2, pp. 134-141. IEEE Computer Society, Los Alamitos (2000)

2. Tao, H., Sawhney, H.S., Kumar, R.: Object tracking with Bayesian estimation of dynamic layer representations. IEEE Transactions on Pattern Analysis and Machine Intelligence 24(1), 75-89 (2002)

3. Weiss, Y., Adelson, E.H.: A unified mixture framework for motion segmentation: Incorporating spatial coherence and estimating the number of models. In: Proceedings of the International Conference on Computer Vision and Pattern Recognition, pp. 321-326. IEEE Computer Society, Los Alamitos (1996)

4. Wang, J.Y.A., Adelson, E.H.: Layered representation for motion analysis. In: Proceedings of the International Conference on Computer Vision and Pattern Recognition, pp. 361-366. IEEE Computer Society, Los Alamitos (1993)

5. Stauffer, C., Grimson, W.E.L.: Adaptive background mixture models for real-time tracking. In: Proceedings of the International Conference on Computer Vision and Pattern Recognition, vol. 2, p. 252 (1999)

6. Stauffer, C., Grimson, W.E.L.: Learning patterns of activity using real-time tracking. IEEE Transactions on Pattern Analysis and Machine Intelligence 22(8), 747-757 (2000)

7. Felzenschwalb, P.F., Huttenlocher, D.P.: Pictorial structures for object recognition. International Journal of Computer Vision 61(1), 55-79 (2005)

8. Korč, F., Hlaváč, V.: Detection and tracking of humans in single view sequences using 2D articulated model. In: Human Motion, Understanding, Modelling, Capture and Animation, vol. 36, pp. 105-130. Springer, Heidelberg (2007)

9. Comaniciu, D., Ramesh, V., Meer, P.: Kernel-based object tracking. IEEE Transactions on Pattern Analysis and Machine Intelligence 25(5), 564-575 (2003)

10. Babu, R.V., Pérez, P., Bouthemy, P.: Robust tracking with motion estimation and local kernelbased color modeling. Image and Vision Computing 25(8), 1205-1216 (2007)

11. Georgescu, B., Comaniciu, D., Han, T.X., Zhou, X.S.: Multi-model component-based tracking using robust information fusion. In: Comaniciu, D., Mester, R., Kanatani, K., Suter, D. (eds.) SMVP 2004. LNCS, vol. 3247, pp. 61-70. Springer, Heidelberg (2004)

12. Jepson, A.D., Fleet, D.J., El-Maraghi, T.F.: Robust online appearance models for visual tracking. IEEE Transactions on Pattern Analysis and Machine Intelligence 25(10), 1296-1311 (2003)

13. Grabner, H., Grabner, M., Bischof, H.: Real-time tracking via on-line boosting. In: Proceedings of the British Machine Vision Conference, vol. 1, pp. 47-56 (2006)

14. Collins, R., Liu, Y., Leordeanu, M.: Online selection of discriminative tracking features. IEEE Transactions on Pattern Analysis and Machine Intelligence 27(10), 1631-1643 (2005)

15. Kristan, M., Pers, J., Perse, M., Kovacic, S.: Closed-world tracking of multiple interacting targets for indoor-sports applications. Computer Vision and Image Understanding (in press, 2008)

16. Ramanan, D.: Learning to parse images of articulated bodies. In: Schölkopf, B., Platt, J., Hoffman, T. (eds.) Advances in Neural Information Processing Systems, pp. 1129-1136. MIT Press, Cambridge (2006)

17. Ramanan, D., Forsyth, D.A., Zisserman, A.: Tracking people by learning their appearance. IEEE Transactions on Pattern Analysis and Machine Intelligence 29(1), 65-81 (2007)

18. Boykov, Y., Funka-Lea, G.: Graph cuts and efficient n-d image segmentation. Int. J. Comput. Vision 70(2), 109-131 (2006) 